\title{
Restoration of Fracture Callus in Osteopetrotic (op/op) Mice by Injection of Macrophage Colony-stimulating Factor
}

\author{
Toshitsugu Kawata, Tadashi Fujita, Shinya Kohno, Masato Kaku, Chiyoko Tokimasa and \\ Kazuo TAnNe \\ Department of Orthodontics, Hiroshima University School of Dentistry, 1-2-3 Kasumi, Minami-ku, Hiroshima 734-8553, Japan \\ (Received 24 January 2000; and accepted 6 March 2000)
}

\begin{abstract}
Morphological studies on bone callus after fracture were carried out in normal and osteopetrotic mutant $(o p / o p)$ mice, in which the tibiae diaphysis were subjected to subperiosteal osteotomy. The fracture callus passed through apparently normal stages in normal mice. Normal osteogenesis with woven bone and occasional cartilage islands were observed in the absence of osteoclasts on the bone surface in op/op mice when macrophage colonystimulating factor (M-CSF) was not injected. When M-CSF was injected into the $o p / o p$ mice, the bone fracture callus passed through the healing stages that resembled to the stages in the normal mice. These results indicate that the fracture callus in the $o p / o p$ mice may be secondary to abnormalities in bone resorption.
\end{abstract}

Osteopetrosis in osteopetrotic $(o p / o p)$ mice is due to a deficiency in mature osteoclast, resulting from a lack of production of functional macrophage colony-stimulating factor (M-CSF) (10). Osteopetrotic $(o p / o p)$ mutation mice were first observed in the Jackson Laboratory in 1970. Kodama et al. (6) reported that an injection of purified recombinant human M-CSF elicited osteoclast differentiation and cured bone sclerosis and failure of tooth eruption in mutant mice. Osteopetrosis is characterized by general accumulation of bone that results in higher risk of bone fracture. Two types of osteopetrosis have been described. The first type, so-called malignant, appears at birth, is an autosomal recessive condition, and is generally lethal. The second is autosomal dominant, appears in adolescence, and is generally more benign. Long bone fractures are common in osteopetrosis, with a lifetime preva-

Correspondence to: Dr T. Kawata, at the above address.

Tel: +81-82-257-5686 Fax: +81-82-257-5687

E-mail: tenzan@hiroshima-u.ac.jp lence of one to ten fractures per patient with the congenital form of the condition (2).

Morphological studies on bone callus after fracture were carried out in normal and osteopetrotic mutant $(o p / o p)$ mice, in which the tibiae diaphysis were subjected to subperiosteal osteotomy.

Mice. Osteopetrotic (op/op) mice and control littermate mice (normal) were obtained from B6C3- $a / a-o p /+$ breeding pairs (Jackson Laboratory, Bar Harbor, ME, USA). Newborn male mice were kept with their mothers, and $o p / o p$ mice were distinguished from normal mice by monitoring eruption of the incisors 11 days after birth. The study was approved by the Animal Use Committee of Hiroshima University, and the animals were maintained in accordance with the guidelines for the care and use of laboratory animals at Hiroshima University.

Surgical procedures. The operated mice were anesthetized via $i . \quad p$. injection of sodium pentobarbital $(0.06 \mathrm{mg} / \mathrm{g}$ body weight). A small incision was then made in the muscle of tibiae under sterile conditions and the soft tissues were 
elevated. The tibiae diaphysis were subjected to subperiosteal osteotomy at 8 weeks after birth. An osteotomy was made between the screws using a manual saw. The tibiae were removed from the mice at 10 or 30 days after osteotomy.

Injection of macrophage colony-stimulating factor (M-CSF). Macrophage colony-stimulating factor of $5 \mu \mathrm{g}$ (rhM-CSF; Austral Biologicals Co., California, USA) was injected into mice 1, 5 , 10, 15, 20 and 25 days after osteotomy.

Light microscopic observations. The tibiae removed from legs were fixed with $4 \%$ formaldehyde for $12 \mathrm{~h}$ at $4{ }^{\circ} \mathrm{C}$, decalcified in $5 \%$ ethylenediamine tetraacetic acid (EDTA; $\mathrm{pH}$ 7.4) for one week, embedded in paraffin, and cut into longitudinal sections of $7 \mu \mathrm{m}$ thickness. These sections were stained for tartrate-resistant acid phosphatase (TRAP), a generally acknowledged cytochemical marker of osteoclasts, and counterstained with hematoxylin. The number of TRAPpositive cells was enumerated on the longitudinal section of the tibiae for uninjected $o p / o p$ mice, injected $o p / o p$ mice and controls.

At 15 days after osteotomy, tissue healing was observed. The fracture callus passed through apparently normal stages in normal mice (Fig. 1a). M-CSF uninjected op/op mice, fracture callus area were smaller than the normal mice (Fig. la and $b)$.

In the bones of normal mice, the bone trabeculae in the diaphysis were mostly replaced by bone marrow showing active hemopoiesis (Fig. 2a). In the op/op mice, the tibiae were still composed of trabecular bone tissue (Fig. 2b). Osteopetrosis is characterized by systemic bone sclerosis and cranial deformation due to defective
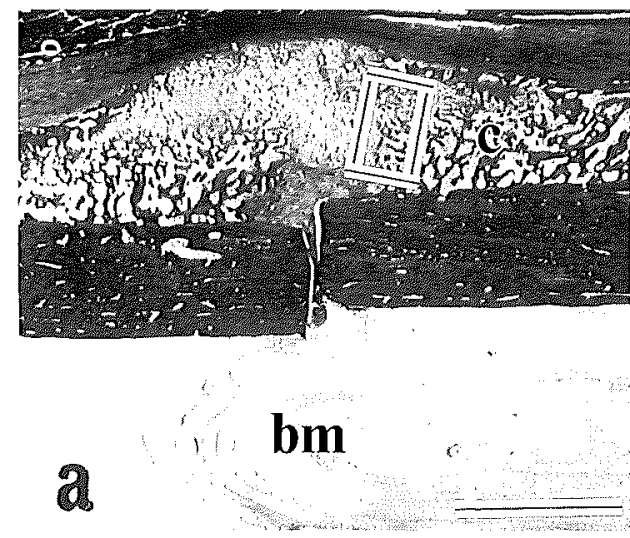

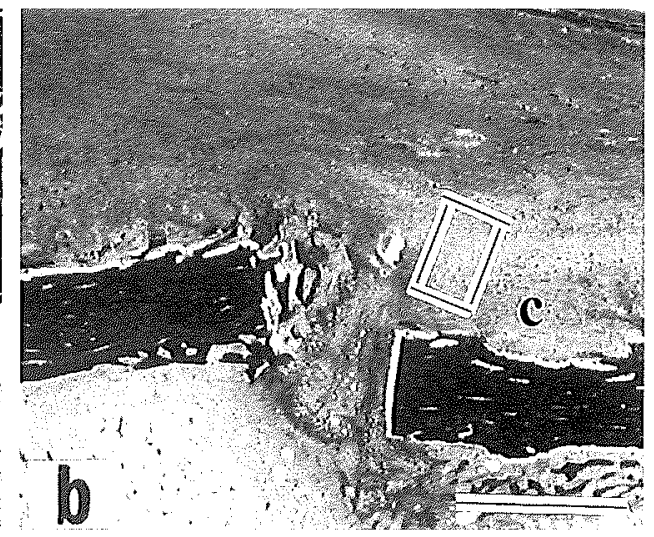

Fig. 1 Photomicrographs of the longitudinal sections at 15 days after osteotomy in normal (a), or op/op (b) mice. A part of the box was observed for light microscopic observations. C; fracture callus, bm; bone marrow (HE stain, horizontal bar $=500 \mu \mathrm{m}$ )

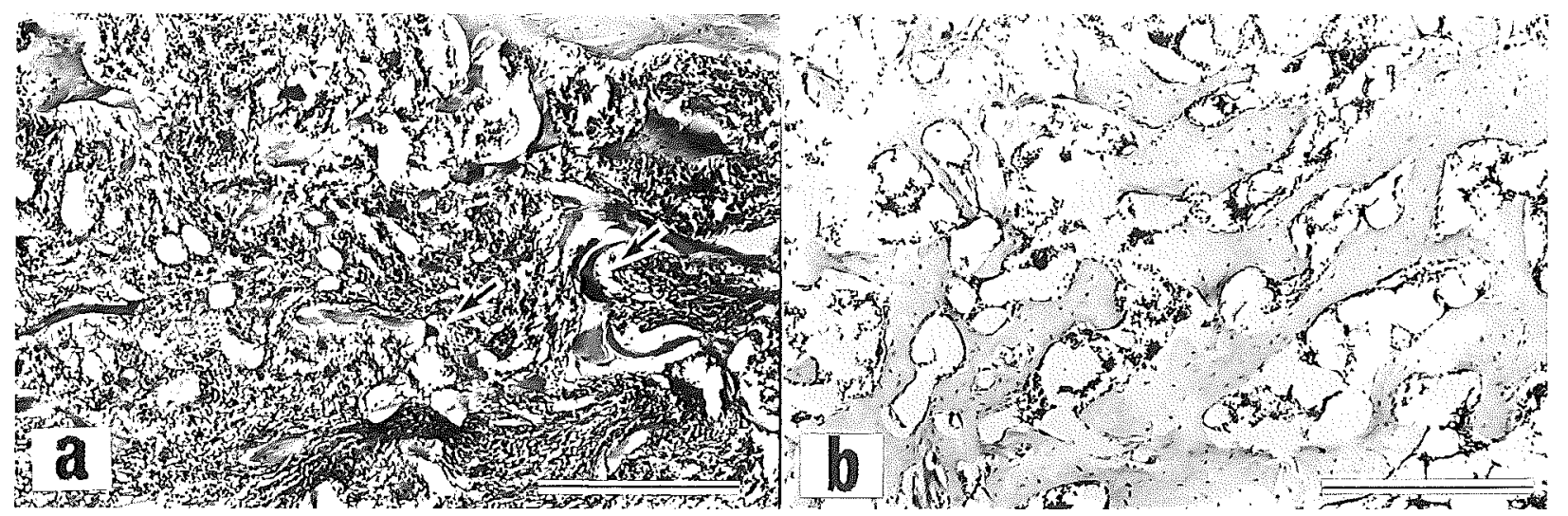

Fig. 2 Photomicrographs of tibiae of $+/$ ? (a) and op/op mice (b). Arrows indicate TRAP-positive osteoclasts.(horizontal bar $=150 \mu \mathrm{m}$ ) 
bone resorption. Previous studies showed that the deficiency of osteoclasts in $o p / o p$ mice was derived from a defect in the production of functional macrophage colony-stimulating factor (M-CSF) $(3,9,10)$. Therefore, lack of bone resorption and remodeling is considered to result from scarcity of osteoclasts in mutant mice (7).

At 10 days after osteotomy, tissue healing was observed in all mice. The defect had been bridged by fracture callus, and much cartilage was observed in the uninjected $o p / o p$ mice (Figs. 3c). Occasional cartilage islands in the uninjected $o p /$ $o p$ mice had more vessels than in normal and injected $o p / o p$ mice (Figs. $3 \mathrm{a}, \mathrm{c}$ and e). Formation of the occasional cartilage islands seemed to take place after the normal stages of fracture callus formation in all groups. However, the fracture calluses of normal mice were bigger than in the uninjected $o p / o p$ and injected $o p / o p$ mice (data not shown). The presence of chondrocytes in osteopetrotic bone may be causally related to the presence of the fracture callus, or may represent chondral metaplasia (5). In the uninjected $o p / o p$ mice, the cartilage layer and the retardation of calcification in it were observed (Figs. 3c and d). Osteocyte lacunae were often empty. The normal and injected $o p / o p$ mice had spaces separating the osteons and the cement lines (Figs. 3b and f). A small number of TRAP-positive sites in the normal and injected $o p / o p$ mice were found exclusively on the outer of the fracture callus surfaces. On the other hand, TRAP-positive cells were observed on the fracture callus surfaces of the uninjected op/op mice. In osteopetrosis, resor-

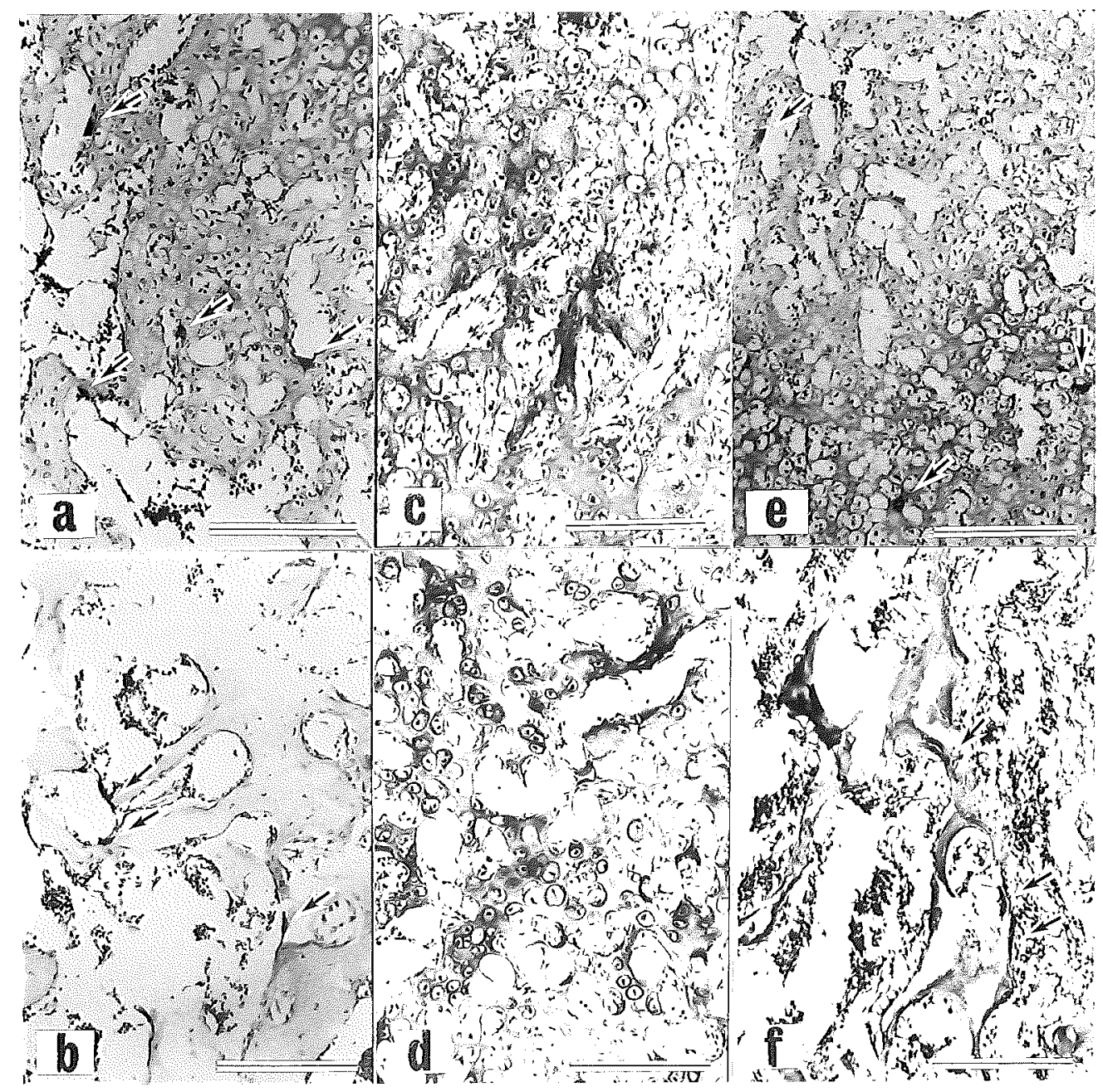

Fig. 3 Photomicrographs of tibiae at 10 days after fracture in normal (a), op/op (c) and M-CSF injected (e) mice; and at 30 days after fracture in normal (b), op/op (d) and M-CSF injected (f) mice. TRAP activity counterstained with hematoxylin. Arrows indicate osteoclasts.(horizontal bar $=200 \mu \mathrm{m}$ ) 
ption is normal, or at least is incomplete, with an altered time-space sequence of events (8). In the 10 days after osteotomy in injected op/op mice, no major changes were observed in comparison with the histological and morphological features in the normal mice.

In the 30 days after osteotomy, the fracture calluses of the normal and injected $o p / o p$ mice gradually became small (data shown). In the op/ $o p$ mice, no major changes were observed in comparison with size of the fracture callus in the 10 days after fracture. In the uninjected op/op mice, the fracture calluses were still composed of cartilage tissue 30 days after osteotomy (Fig. 3d). On the other hand, the osteoclast deficiency in $o p / o p$ mice can be cured (6), and the fracture callus was restored by injection of M-CSF (Fig. 3f).

The features of bone remodeling in $o p / o p$ mice are controversial. According to some authors, bone remodeling occurs normally but is rather slow in M-CSF uninjected op/op mice (4). By remodeling, normal bone is able to reduce the stresses concentrated in microfractures and macroscopic defects. This does occur in osteopetrotic bone, and these micro- and macroscopic defects tend to propagate along areas of lower resistance, such as cement lines, lacunae, and Haversian canals (1). When M-CSF was injected into the $o p / o p$ mice, the bone fracture callus passed through the healing stages that resembled to the stages in the normal mice. These results indicate the fracture callus in the op/op mice may be secondary to abnormalities in bone resorption.

\section{Acknowledgments}

The authors are grateful to the Research Facilities for Laboratory Animal Science, Hiroshima Uni- versity School of Medicine.

\section{REFERENCES}

1. Carter D. R. and Hayes W. C.(1977) Compact bone fatigue damage: a microscopic examination. Clin. Orthop. 127, 265-274.

2. Dahl N., Holmgren G., Holmberg S. and Esmark H.(1992) Fracture patterns in malignant osteopetrosis (AlbersSchonberg disease). Arch. Orthop. Trauma Surg. 111, 121-123,

3. Felix R., Cecchini M. G., Hofstetter W., Elford P. R., Stutzer A. and Freisch H (1990) Impairment of macrophage colony-stimulating factor production and lack of resident bone marrow macrophages in the osteopetrotic op/op mouse. J. Bone Miner. Res. 5, 781-789.

4. Kaku M., Kawata T., Kawasoko S., Fujita T., Tokimasa C., Niida S. and Tanne K.(1998) Osteoclast appearance in developmental stages of different bones after a single injection of macrophage colony-stimulating factor in $o p /$ op mice. Bioned. Res. 19, 77-81.

5. Kirkpatrick Jr. J. A.(1968) Orthopedic disease. Physiology, pathology, radiology. WB Saunders Company, Philadelphia, p. 161-169.

6. Kodama H., Yamasaki A., Nose M., Niida S., Ohgame Y., Abe M., Kumegawa M. and Suda T.(1991) Congenital osteoclast deficiency in osteopetrotic $(o p / o p)$ mice is cured by injections of macrophage colony-stimulating factor. $J$. Exp. Med. 173, 269-272.

7. Marks S. C. Jr. and Cahill D. R.(1987) Regional control by the dental follicle of alterations in alveolar bone metabolism during tooth eruption. J. Oral Pathol. 16, 164-169.

8. Shapiro F., Glimcher M. J., Holtrop M. E., Tashjian A. H. Jr., Brickley-Parsons D. and Kenzora J. E.(1980) Human osteopetrosis: a histological, ultrastructural, and biochemical study. J. Bone Joint Surg. Am. 62, 384-399.

9. Wiktor-Jedrzejczak W., Bartocci A., Ferrante A. W., Ahmed-Ansari A., Sell K. W., Pollard J. W. and Staley E. R.(1990) Total absence of colony-stimulating factor 1 in the macrophage-deficient osteopetrotic $(o p / o p)$ mouse. Proc. Natl. Acad. Sci. USA 87, 4828-4832.

10. Yoshida H., Hayashi S., Kunisada T., Ogawa H., Nishikawa S., Okamura H., Sudo T., Shutz L. D. and Nishikawa S.(1990) The murine mutation osteopetrosis is in the coding region of the macrophage colony stimulating factor gene. Nature 345, 442-444. 